\title{
SURGICAL COMPLICATIONS OF PARAPHARYNGEAL SCHWANNOMA: OUR
} EXPERIENCE

Dr Debabrata Das

MBBS. MS. Assistant Professor. Department of ENT and Head Neck Surgery. R G Kar Medical College and Hospital. Kolkata. 700004

Dr Pritha Ghosh MBBS. MS. Senior Resident. Department of ENT and Head Neck Surgery. Ranaghat Sub Divisional Hospital. Nadia. 741201

Dr Riya Das

MBBS. MS Post Graduate Trainee. Junior Resident. Department of ENT and Head Neck Surgery. R G Kar Medical College and Hospital. Kolkata. 700004

\section{Dr Tanmoy} Sarkar*

MBBS. MS Post Graduate Trainee. Junior Resident. Department of ENT and Head Neck Surgery. R G Kar Medical College and Hospital. Kolkata. 700004 *Corresponding Author

\section{ABSTRACT}

Introduction-Tumors of parapharyngeal space are rare. Schwannomas, originating either from Schwann cells or fibroblasts supporting the nerve, are one of the benign neurological tumors occurring in this space. Surgical excision is the mainstay of its management. Transcervical approach is carried out for most of the parapharyngeal schwannomas. This article aims to study the clinical and radiological presentation of parapharyngeal schwannoma and also to study post-operative neurological outcome. Materials and methods - A retrospective study was conducted from June 2018 to November 2019 (1.5 years) at the Department of Otolaryngology, in a tertiary care medical college \& hospital. A total of 7 cases of parapharyngeal schwannoma were selected; the clinical, radiological presentation was studied. Post-operative complications were studied within a follow up period of 8 months. Results - The mean age of presentation was 44 years. Male: Female ratio was 5:2. Five patients $(71.4 \%)$ presented with left sided lesions whereas 2 patients $(28.6 \%)$ were with right sided lesions. The most common presentation was an asymptomatic palpable neck mass in 4 patients (57.1\%). 4 patients (57.1\%) had mass arising from vagus nerve, 2 patients $(28.6 \%)$ had mass arising from sympathetic trunk and one patient $(14.3 \%)$ had mass arising from hypoglossal nerve. 4 patients $(57.1 \%)$ had neurological complication in immediate post-operative period, out of which 3 patients (42.8\%) improved within 8 months follow up. Conclusion-Most of the parapharyngeal schwannoma presents with asymptomatic slow growing neck and/or oropharyngeal mass. Progressive growth can result in dysphagia. Surgical excision may accompany neurological complications. Outcome of neurological sequelae depends on preserving the anatomical integrity of the nerve from which the tumor arises.

\section{KEYWORDS}

parapharyngeal tumors; nerve; transcervical approach; complications; neurological

\section{INTRODUCTION:}

Parapharyngeal space (PPS) is an inverted pyramid shaped deep neck space extending superiorly from skull base to inferiorly at the greater cornu of the hyoid bone. Parapharyngeal space tumors account for $0.5 \%$ of all head and neck masses. ${ }^{[1]}$ A number of differential diagnosis can be there into the view of PPS tumors, out of them tumors of salivary gland origin being the commonest. ${ }^{[2]}$ Schwannomas account for some rare occurrence in the parapharyngeal space arising mainly from the sympathetic trunk and from last four cranial nerves.

The PPS is divided by fascial condensation called aponeurosis of Zukerkandl and Testut (fascia joining the styloid process to the tensor veli palatini) into the pre-styloid space and the post-styloid space $\mathrm{e}^{[3]}$

The PPS nerve sheath tumors are predominantly treated by surgery. External transparotid, transcervical approaches have traditionally been considered the only safe access. ${ }^{[3]}$ The choice of surgical technique is dependent on the tumor size, its relationship to the skull base, relationship to the parotid gland and malignancy potential of the tumor. The transcervical approach is most commonly performed approach for PPS tumors. ${ }^{[4]}$

In our study, all the patients of PPS schwannoma underwent surgery through transcervical route. The 7 patients had mass in the cervical region, none of them extending high up to the skull base. Most common post operative complication was cranial nerve $(\mathrm{CN})$ palsy. We studied 7 patients of PPS schwannoma among which 4 patients had major post-operative complications. $\mathrm{CN}$ palsy was encountered most and there outcome was carefully observed in this study. Though most of them recovered in due course of time, but a case of hypoglossal schwannoma did not improve till date.

\section{Aims:}

1. To study the clinical presentation of parapharyngeal tumor.

2. To study the radiological characteristics.

3. To study the surgical complications.

\section{Methodology:}

Study design: Retrospective observational analytical study
Total number of cases: 7 patients.

Study setting and time period: The study was conducted in the department of Otoloaryngology and Head Neck Surgery in a tertiary Medical College and Hospital June 2018 to November 2019 (1.5 years).

Inclusion criteria: Patients who were diagnosed as PPS nerve sheath tumor or schwannoma.

Exclusion criteria: PPS tumors other than schwannoma.

Pre-operative protocol: All the patients underwent detailed clinicoradiological assessment which includes CT scan, MRI \& FNAC. Idea about the origin of nerve sheath schwannoma was obtained from the radiological investigations. Carotid artery angiography was done in one patient wherein confusion was raised between carotid body tumor and vagal schwannoma. After taking informed consent they were selected for operative procedure after anaesthetic optimization.

Surgical method: All the patients underwent surgery through the transcervical approach under general anesthesia with the patient intubated with a nasotracheal tube [Fig 3]. The patients were positioned with the neck extended and rotated opposite to the side of the lesion. A transverse skin incision was made approximately $5 \mathrm{~cm}$ below the lower border of the mandible to spare the marginal mandibular branch of facial nerve. After incising platysma and raising superior flap in subplatysmal plane, the submandibular gland was mobilized in most of the cases to get greater access to the parapharyngeal space. External jugular vein, greater auricular nerve were identified over the sternocleidomastoid muscle. The mass was palpated after retracting the sternocleidomastoid muscle posteriorly. Blunt finger dissection was performed once reaching to the intracapsular plane preserving the sheath of the nerve as much as possible. After tumor removal and achieving adequate hemostasis a negative suction drain was placed in all the patients and wound was closed in layers. Upper carotid exposure was not needed in any of the 
cases as the mass was in cervical region in all patients.

Follow up: The patients were followed up on post-operative day 7, day 14 , first month, third month, sixth month and eighth month. After eight months the patients were evaluated regarding the post-operative neurological sequels.

\section{RESULTS:}

Total 7 patients were studied in a retrospective way. Out of them 5 patients were female and 2 patients were male. So the female patients (71.4\%) outnumbered the male patients $(28.6 \%)$. Maximum number of patients $(57.1 \%)$ was in age group of $41-50$ years. 5 patients $(71.4 \%)$ had left sided lesion, whereas 2 patients $(28.6 \%)$ had mass in right side of neck. 4 patients $(57.1 \%)$ presented with palpable mass in neck, 3 patients $(42.8 \%)$ presented mainly with visible oropharyngeal bulge [Fig 1]. 3 patients complained about dysphagia due to oropharyngeal bulge.

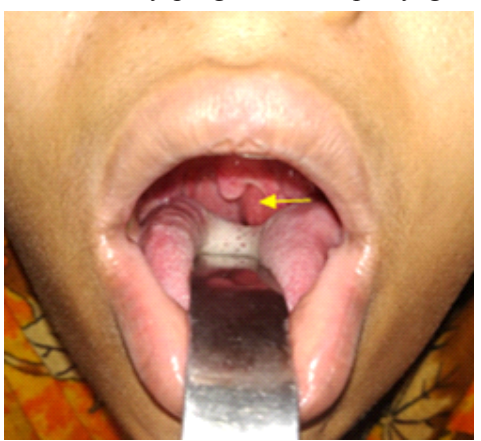

In all the cases the FNAC were suggestive of nerve sheath tumor or schwannoma. Contrast enhanced CT scan revealed that all the tumors arised from the post-styloid compartment. MRI scan showed that 4 patients had mass within the carotid sheath, most of them separating great vessels i.e. carotid artery and jugular vein [Fig 2] but none of them were hypervascular or heterogenous. Rest of the 3 patients had mass outside the carotid sheath. 2 patients had mass which pushed the carotid artey and jugular vein antero-laterally, most probably the nerve of origin was cervical sympathetic chain. All the tumors were excised through transcervical route under general anesthesia. We had to ligate external carotid artery in one patient of vagal schwannoma to access a large PPS tumor. Pre-operatively none of the patients had any neurological deficit. Intra-operatively it was found that 4 patients $(57.1 \%)$ had mass arising from vagus nerve, 2 patients $(28.6 \%)$ had mass arising from the cervical sympathetic chain and one patient $(14.3 \%)$ had mass arising from hypoglossal nerve. Post-operative histopathological examination was done in all the excised masses which also indicated schwannoma.

One tumor was arising from the hypoglossal nerve which was seen intra-operatively [Fig 3]. This patient had hypoglossal nerve palsy in immediate post-operative period. Hypoglossal nerve palsy was detected by deviation of tongue to opposite side. One patient of vagal schwannoma had glossopharyngeal nerve palsy which was improved by $3^{\text {rd }}$ month in post-operative period but the hypoglossal nerve palsy did not improve till date. One patient had ipsilateral horner's syndrome in immediate post-operative period which were improved too after 3 months. One patient of vagal schwannoma had ipsilateral vocal cord paresis in immediate post-op period which improved by $6^{\text {th }}$ postoperative month.

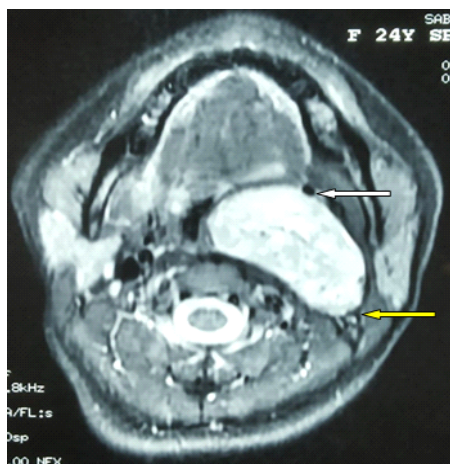

Fig 2: PPS mass splaying the carotid artery (white arrow) and jugular vein (yellow arrow)

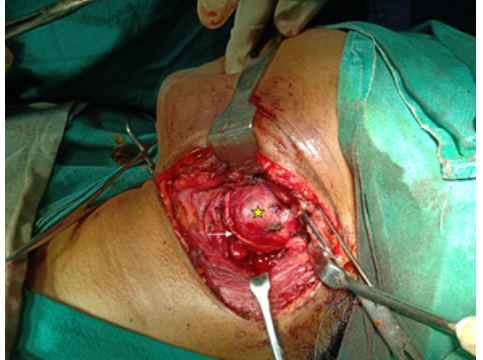

Fig 3: Mass arising from the hypoglossal nerve through transcervical approach. Yellow asterisk- PPS mass. White arrowHypoglossal nerve.

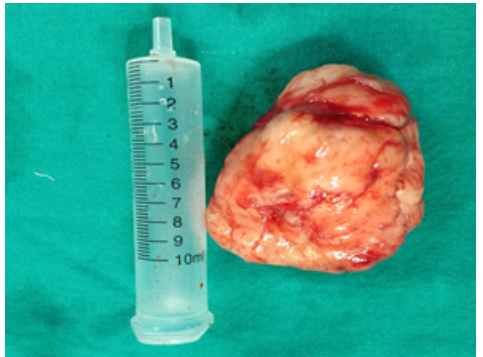

Fig 4: Avagal schwannoma mass after removal

\section{DISCUSSION:}

Schwannomas are benign neurogenic tumors considered to originate from either Schwann cells or fibroblasts supporting the nerve. PPS schwannoma patients most commonly present between the ages of 30 and 70 years. ${ }^{[5]}$ Most of these uncommon tumors affect the vagus nerve and the cervical sympathetic chain. ${ }^{[6]}$ FNAC should be done to rule out other differential diagnosis of PPS tumor. The pre-operative CT scan and MRI can determine the diagnosis ${ }^{[7]}$ and may indicate the nerve of origin. ${ }^{[8]} \mathrm{CT}$ scan can differentiate between pre-styloid and post-styloid mass. Contrast enhanced CT scan is helpful to know the relation of the tumor with the great vessels i.e common carotid artery, external and internal carotid artery and the internal jugular vein. In our study 2 patients had mass which pushed the great vessels antero-laterally without splaying of internal and external carotid arteries. So preoperatively it helped us to predict if the mass originated from cervical sympathetic chain. MRI allows for superior soft tissue contrast resolution and avoids exposure of the patient to ionizing radiation. For these reasons, MRI is now considered the imaging study of choice to evaluate parapharyngeal space tumors. ${ }^{[9]}$ In contrast to paraganglioma, there are no vascular flow voids seen in schwannoma. The vagal schwannomas (4 patients) were differentiated from carotid body tumor on basis of MRI though all of them showed separating the great vessels i.e carotid artery and jugular vein to some extent. In MRI the hypervascular lesions like carotid body tumor will present as 'salt and pepper appearance' at the bifurcation of external and internal carotic artery. MR angiography was done in one patient in our study to rule out enhancing vascular lesion and to differentiate from carotid body tumor. In our case series, all the 7 patients had mass lying in poststyloid compartment. Three patients had mass outside carotid sheath.

Complete surgical resection remains the treatment of choice for PPS schwannoma as the tumors are relatively radioresistent. ${ }^{[5],[10]}$ In our study, we carried out transcervical approach in all our patients. Superior flap was elevated in the subplatysmal plane upto the angle of mandible, and inferior flap upto the level of thyroid notch. After reaching the plane of the mass, the hypoglossal schwannoma was seen as well encapsulated yellowish mass arising from the hypoglossal nerve sheath whereas the masses originated from sympathetic chain was seen outside the carotid sheath, sometimes pushing the great vessels laterally or anteriorly or anterolaterally. The sympathetic chain was identified after identifying the carotid artery and internal jugular vein and retracting them laterally. Intraoperatively the vagal schwannomas were seen as masses originating from the vagus nerve within the carotid sheath. Meticulous dissection was carried out to dissect the mass from the great vessels and also to dissect the mass free from vagus nerve sheath. Incision was given over the nerve sheath along the axis of the nerve and intracapsular dissection was done all along with all effort to preserve the nerve sheath as much as possible. We avoided electrocautery at the time of the intracapsular dissection. 
Manipulation was done as gentle as possible and pulse, blood pressure were monitored closely specially in case of vagal schwannoma with the help of anaesthesiology team. Surgicel was used to achieve haemostasis in perineural sites. Hypoglossal nerve was identified after retracting the submandibular gland deep to the posterior belly of digastrics muscle and running forward as a horizontal structure and the mass was originating from the sheath of this nerve [fig 3]. The large mass was extending from skull base to the PPS. Manipulation of the mass caused significant stretching of hypoglossal nerve along with vascular compromise. Most probably this was the reason for permanent hypoglossal nerve paralysis.

Because schwannomas originate from either Schwann cells or fibroblasts supporting the nerve, they tend to compress the nerve fascicles as they enlarge and can usually be dissected free of all structures except the parent nerve fascicle.$^{[10],[12]}$ Most of the time the involved nerve pass over the tumor capsule. ${ }^{[5]}$ In most cases, patients should be prepared for dysfunction of the involved nerve after schwannoma resection though literature reports few cases who didn't have neurological dysfunction post-operatively. ${ }^{[5]}$ In our study, though 4 patients among the 7 patients had major post-operative neurological deficit but only one patient with hypoglossal nerve injury had permanent neuropathy in 8 months follow up period. As in most of the cases anatomical integrity of the nerves were well maintained, unnecessary stretching were avoided also vascular integrity was well maintained, the neurological deficits were temporary in immediate post-operative period and they revived in long term follow up period.

All the patients were examined pre-operatively for any neurological dysfunction. Fibreoptic laryngoscopy was done to rule out laryngeal nerve palsy. None had any neurological dysfunction. In post operative period, one patient had hypoglossal and another patient had glossopharyngeal nerve palsy. The glossopharyngeal nerve palsy which later improved was most probably associated with blunt dissection of the tumor to free it from tumor bed in a patient of vagal schwannoma. One patient had ipsilateral Horner's syndrome in postoperative period which improved after 3 months. One patient of vagal schwannoma who had ipsilateral vocal cord palsy recovered after 6 months.

\section{CONCLUSION:}

Schwannomas are the most common neurogenic tumor in parapharyngeal space. ${ }^{[2]}$ Surgical excision is the mainstay treatment of PPS schwannoma but surgical excision may results in postoperative neurological deficit. CT scan and MRI were done in all patients to know the site, size and extension of the tumor and also to get an idea about the nerve of origin. ${ }^{[8]}$ This helped the surgeon to predict the expected neurological deficit preoperatively and counsel the patient accordingly and also to plan the surgery.

4 out of our 7 patients developed neurological deficit in the immediate post operative procedure but as the anatomical integrity of the nerve were preserved in most of the cases; they recovered after a certain period of time.

\section{REFERENCES:}

1. Khafif Avi, Segev Yoram, Kaplan Daniel M, Gil Ziv, and Fliss Dan M, "Surgical management of parapharyngeal space tumors: a 10-year review," Otolaryngology — Head and Neck Surgery, vol. 132, no. 3, pp. 401-406, 2005.

2. Riffat F, Dwivedi RC, Palme C, et al. A systematic review of 1143 parapharyngeal space tumors reported over 20 years. Oral Oncol 2014; 50(5):421-30.

3. Watkinson J. C. (Ed.), Clarke R. W. (Ed.). (2018). Scott-Brown's Otorhinolaryngology Watkinson J. C. (Ed.), Clarke R. W. (Ed.). (2018). Scott-Brown's Otorhinolaryngology
and Head and Neck Surgery. Vol 3. Page- 163. Boca Raton: CRC Press, https://doi.org/10.1201/9780203731000.

4. Basaran B, Polat B, Unsaler S, Ulusan M, Aslan I, Hafiz G. Parapharyngeal space tumours: the efficiency of a transcervical approach without mandibulotomy through review of 44 cases. Acta Otorhinolaryngol Ital. 2014;34(5):310-316.

5. Chang SCSchi YMNeurilemmoma of the vagus nerve: a case report and brief literature review. Laryngoscope 1984;94 (7) 946-949

6. Mikaelian DOHolmes WFSimonian SK Parapharyngeal schwannomas. Otolaryngol Head Neck Surg 1981;89(1) 77-81

7. Gopinathan Anil and Tiong-Yong Tan. CT and MRI evaluation of nerve sheath tumors of the cervical vagus nerve. American Journal of Roentgenology 2011; 197: 195-201.

8. Saito DM, Glastonbury CM, El-Sayed IH, Eisele DW. Parapharyngeal Space Saito DM, Glastonbury CM, El-Sayed IH, Eisele DW. Parapharyngeal Space
Schwannomas: Preoperative Imaging Determination of the Nerve of Origin. Arch Otolaryngol Head Neck Surg. 2007;133(7):662-667. doi:10.1001/archotol.133.7.662

9. Miller FR, Wanamaker JR, Lavertu P, Wood BG. Magnetic resonance imaging and the management of parapharyngeal space tumors. Head Neck. 1996;18(1):67-77. doi:10.1002/(SICI) 1097-0347(199601/02)18:1<67::AID-HED9>3.0.CO;2-X

10. Gilmer-Hill HSKline DG Neurogenic tumors of the cervical vagus nerve: report of four cases and review of the literature. Neurosurgery 2000;46 (6) 1498-1503

11. Kuet ML, Kasbekar AV, Masterson L, Jani P. Management of tumors arising from the parapharyngeal space: a systematic review of 1,293 cases reported over 25 years. Laryngoscope 2015;125(6): 1372-81.

12. Wax MKShiley SGRobinson JLWeissman JL Cervical sympathetic chain schwannoma. Laryngoscope 2004;114(12) 2210-2213

13. Som PMSacher MStollman ALBiller HFLawson W Common tumors of the parapharyngeal space: refined imaging diagnosis. Radiology 1988;169(1) 81-85 